\title{
Trastornos específicos del desarrollo del lenguaje: bases neurobiológicas
}

\author{
J. Narbona-García, E. Schlumberger \\ Unidad de Neurología Pediátrica. Dpto. de Pediatría. Clínica Universitaria de Navarra. \\ Facultad de Medicina. Universidad de Navarra. Pamplona, España.
}

\section{RESUMEN}

En el origen de las disfasias o trastornos específicos del desarrollo del lenguaje (TEDL) se postula actualmente una predisposición genética, evidenciada por estudios de gemelos, de árboles familiares y de síndromes peculiares; tal predisposición sigue la mayor parte de las veces el modelo multifactorial pero también en algunas familias se demuestra una transmisión autosómica dominante de incompetencia para distintos componentes modulares del lenguaje. Los estudios de neuroimagen pueden mostrar alteraciones de la asimetría volumétrica entre los hemisferios cerebrales. Un grupo no pequeño de TEDL está en relación fisiopatológica con actividad epileptiforme durante la vigilia y el sueño no-REM, y el tratamiento antiepiléptico, sobre todo con esteroides; se ha mostrado útil en estos casos. Los sujetos con TEDL tienen una especial dificultad para la discriminación fina de índices temporales y ello invita a establecer una hipótesis de trabajo que tenga en cuenta una posible disfunción de estructuras subcorticales (talamoestriadas y cerebelosas) que probablemente modulan el trabajo de la corteza en la integración fonológica.

\section{PALABRAS CLAVE}

Discriminación temporal. Disfasia. Epilepsia. Genética. Trastorno del desarrollo del lenguaje.

Correspondencia: Dr. Juan Narbona-García. Clínica Universitaria de Navarra.

Avda. Pío XII, s/n. E-31080 Pamplona. 


\section{INTRODUCCIÓN}

Existe un grupo de dificultades en el desarrollo del lenguaje que no pueden ser suficientemente justificadas por defectos instrumentales auditivos o de mecánica fonoarticulatoria ni por retraso intelectual, ni por otros trastornos psicopatológicos o deprivación socio-afectiva [1-9]. Se les denomina genéricamente disfasias o trastornos específicos del desarrollo del lenguaje (TEDL) y revisten diversas modalidades clínicas $[4,9,10]$ según la proporción en que estén afectadas las competencias fonológicas, sintácticas y lexicales en las vertientes de comprensión y expresión. A su vez, pueden asociarse a trastornos de la comunicación (espectro autista) o proporcionar el trasfondo de trastornos del aprendizaje, como la dislexia-disortografia.

\section{FISIOPATOLOGÍA DE LOS TEDL}

\section{Anomalías morfológicas y funcionales de la corteza cerebral}

Si se exceptúan un par de estudios necrópsicos de caso único [11,12], que mostraban anomalías displásicas de la corteza perisilviana en el cerebro de sujetos con TEDL y algunas observaciones en cerebros de sujetos disléxicos [13], la gran mayoría de los trabajos morfológicos se han basado en la neuroimagen. Sobre la resonancia magnética cerebral de un grupo de sujetos con TEDL y de un grupo control se halló disminución volumétrica, en el grupo patológico, de las regiones infrasilvianas posteriores en ambos hemisferios y de las regiones infrasilviana anterior y parietal inferior en el hemisferio izquierdo [14]. Estos hallazgos parecen indicar que los hemisferios cerebrales de los sujetos con TEDL, considerados como grupo, presentan una ausencia de la asimetría volumétrica normal, o bien la asimetría está invertida a favor del hemisferio derecho, mientras que en la población general la asimetría interhemisférica favorece al izquierdo [15-17]. Se ha postulado que la testosterona influenciaría, ya desde la vida intrauterina, el diferente crecimiento de los hemisferios, induciendo la maduración del derecho o acelerando la muerte celular programada en el izquierdo [18]. Esta hipótesis parece apoyada por el hecho de que en niños con hiperplasia suprarrenal congénita (afección en la que se genera un exceso de testosterona desde la vida intrauterina, causada por una mutación genética autonómica recesiva), así como en sus hermanos heterocigotos, se observa una alta prevalencia de trastornos del desarrollo del lenguaje y una pérdida o inversión significativas de la asimetría interhemisférica, en comparación con controles de las mismas familias no portadores de la mutación [19].

Mediante SPECT en situación basal, se ha apreciado una hipoperfusión de ambos lóbulos temporales, más acentuada sobre el izquierdo, en un grupo de niños con TEDL y/o trastornos de atención, frente a un grupo control constituido por hermanos sanos de los pacientes [20]. Estudios de flujo cerebral durante la realización de tareas verbales $[21,22]$ mostraron una activación deficiente del hemisferio izquierdo.

Los niños con TEDL muestran una frecuencia significativamente elevada de anomalías paroxísticas uni o bilaterales en el electroencefalograma de vigilia, sin que esto se asocie necesariamente a crisis epilépticas [23,24]. Asimismo, la presencia de descargas paroxísticas generalizadas durante el sueño no-REM es altamente llamativa en estos 
sujetos, llegando a constituirse en algunos casos un estado de punta-onda continua durante el sueño lento [25-27]. Actualmente, se discute la posible relación fisiopatológica entre dichos paroxismos eléctricos cerebrales mantenidos y el origen de los TEDL y de otras patologías como el autismo infantil [24,27-29]. Aunque podría tratarse de una concomitancia de fenómenos clínicos y electroencefalográficos, es plausible que estén fisiopatológicamente relacionados entre sí, lo cual invita a ensayar terapias con fármacos antiepilépticos. Al igual que en el síndrome de Landau-Kleffner, la terapia esteroidea se ha mostrado eficaz en algunos casos de TEDL con actividad paroxística en el EEG de sueño [30], examen que debe formar parte del protocolo de estudio de dichos pacientes [28-30] (Tabla).

\section{Disfunción de estructuras subcorticales}

Los trabajos de Tallal y su equipo durante los últimos veinticinco años [31-35] han mostrado que los niños con TEDL presentan una dificultad innata en el procesamiento de índices temporales (del orden de algunas decenas de milisegundos) ante tareas auditivas verbales y no verbales, así como en las motrices. Por otra parte, la investigación neurofisiológica ha puesto de manifiesto que las conexiones recíprocas talamocorticales generan una actividad oscilatoria en la que predominan los ciclos a 40 Hz. A esta actividad rítmica de los 'relojes cerebrales' se atribuye una función de coalescencia témporo-espacial que, probablemente, posibilita la experiencia consciente y sirve como marco de referencia para el procesamiento de la información sensorial, para la organización de la conducta motora y para el 'esculpido' cerebral desde etapas muy tempranas del desarrollo [36-39]. Respecto al lenguaje, la percepción temporal y la memoria de trabajo son imprescindibles para la consciencia fonológica [40]. Los circuitos talamocorticales generadores de la citada actividad oscilatoria están, a su vez, modulados por los ganglios de la base y por el cerebelo.

Crosson [41], mediante un metanálisis de observaciones anatomoclínicas de afasia subcortical y de abundantes datos de experimentación neurofisiológica, ha propuesto un modelo neurolinguiístico que incluye una red paralela subcortical en la que intervienen el tálamo y los núcleos grises de la base cerebral; la porción anterior del área de Broca estaría conectada, a través de los núcleos ventral anterior y pulvinar del tálamo, con el área de Wernicke. Esta conexión sería bidireccional y permitiría el ajuste semántico de la producción verbal. Por su parte, las citadas estructuras talámicas estarían bajo la influencia inhibitoria del globo pálido que, a su vez, es inhibido por el núcleo caudado, el cual establece conexiones con la corteza. Así, se pone en juego un control córticosubcortical del flujo verbal y de la organización secuencial de los enunciados.

El cerebelo, por su parte, también parece desempeñar una función relevante no sólo en el control motor articulatorio del habla, sino también en la programación fonológicosintáctica y, probablemente, en la recuperación lexical. Las lesiones cerebelosas agudas en niños (vasculares, traumáticas, exéresis tumorales) ocasionan con frecuencia un mutismo que puede persistir durante varias semanas, a continuación se recupera progresivamente $[42,43]$ y presenta entonces características comunes con la afasia transcortical motora. Otra entidad interesante al respecto es la apraxia oculomotora congénita, síndrome genético autosómico recesivo que consiste básicamente en una incapacidad para los movimientos oculares sacádicos, cuya base fisiopatológica parece residir en una disfunción de las conexiones córtico-cerebelosas. En una serie reciente de 
ocho niños con este síndrome, de los cuales todos menos uno mostraban hipoplasia de vermis inferior en la neuroimagen, la mitad de ellos sufrían también grados variables de apraxia verbal [44]. Hemos constatado este mismo hallazgo en la mitad de seis pacientes con apraxia oculomotora congénita y en cinco de once sujetos con ataxia cerebelosa congénita no progresiva (series no publicadas).

\section{PREDISPOSICIÓN GENÉTICA Y FACTORES AMBIENTALES}

El lenguaje, al igual que otras capacidades cognitivas y comportamentales, se desarrolla a partir de unos condicionantes genéticos múltiples que interactúan con los factores de entorno; por ello, es lógico postular que en la causalidad de los TEDL exista, en mayor o en menor grado, una base genopática. Los argumentos en favor de esto provienen principalmente de tres tipos de estudios:

- El análisis de la concordancia para TEDL entre gemelos [45], que es muy superior entre los monocigóticos frente a los dicigóticos y entre éstos frente a los hermanos no gemelos.

- El "estudio de familias con ocurrencia de TEDL en varias generaciones, que ha permitido mostrar, la mayoría de las veces, una predisposición de tipo poligénico [46,47]; si bien en algunas familias se ha observado un patrón autosómico dominante con cierta heterogeneidad de expresión fenotípica [48]. Otros estudios detallados de grupos familiares han conseguido revelar que la anomalía genética - aun sin haberse confirmado molecularmente en ningún caso- se traduce fenotípicamente en una dificultad específica (modular), bien para el desarrollo de la gramática [49] o bien para las habilidades fonológicas [50,51].

- En el fenotipo de algunas entidades nosológicas con deficiencia mental cuyo marcaje genético es bien conocido actualmente, destacan ciertas conductas verbales características. Así, en el síndrome de frágil X se observa déficit mixto fonológico-sintáctico [52]; en el síndrome de Angelman por deleción en el cromosoma 15 o por disomía uniparental (materna) del citado cromosoma, destaca una apraxia verbal, que se corresponde con la existencia de anomalías citoarquitectónicas corticales perisilvianas [53,54]; los sujetos con síndrome de Williams, cuyo marcador genético es una deleción en el locus para el gen de la elastina, presentan conducta hiperverbal con déficit semántico-pragmático, buen nivel lexical, capacidad gramatical sólo ligeramente afectada y torpeza psicomotiz notable $[55,56]$.

El posible papel de ciertos factores ambientales adversos (prematuridad, anoxoisquemia perinatal, desnutrición intrauterina, modelos de lenguaje distorsionado en el entorno familiar, etc.) en la génesis de los TEDL ha sido destacado por varios autores [57-63].

\section{CONSIDERACIONES FINALES}

Hoy sabemos que ciertas lesiones congénitas o posnatales unihemisféricas muy tempranas, por muy voluminoso que sea su aspecto en la neuroimagen, no conllevan, 
por lo general, detrimento notable de las capacidades verbales. El desarrollo lingüístico de los niños con hemiplejía congénita derecha o izquierda, considerados como grupo, es sólo ligeramente inferior al de los sujetos control; si acaso, los hemipléjicos derechos (lesión hemisférica izquierda) desarrollan una sintaxis y un vocabulario sólo moderadamente pobre respecto a los hemipléjicos izquierdos [64, 65]. Sorprende la levedad general de las anomalías del lenguaje - cuando existen- en estos casos con lesiones cerebrales muy evidentes, frente a la práctica ausencia de anomalías neurológicas mayores en los casos de TEDL, así como en los trastornos del espectro autista. Por ello parece útil tener también en cuenta las citadas estructuras subcorticales y su papel, con la corteza cerebral, en la discriminación temporal; una disfunción temprana a este nivel puede explicar el origen de un grupo importante de trastornos del desarrollo del lenguaje [34, 35, 66, 67].

\section{BIBLIOGRAFÍA}

1. Benton AL. Developmental aphasia and brain damage. Cortex 1964; 1: 40-52

2. Wilson BC, Risucci DA. A model for clinical quantitative classification. Generation. I. Application to language disordered preschool children. Brain Lang 1986; 27: 281-309.

3. Tuchman RF, Rapin J, Shinnar S. Autistic and dysphasic children. I. Clinical characteristics. Pediatrics 1991; 88: 1211-8.

4. Rapin I, Allen DA, Dunn MA. Developmental language disorders. In Segalowitz SJ, Rapin I, eds. Handbook of neuropsychology. Child Neuropsychology. Vol 7. Amsterdam: Elsevier Science; 1992. p. 111-38.

5. Bishop DVM. The biological basis of specifíc language impairment. In Fletcher C, Hall D, eds. Specific speech and language disorders in children. London: AFASIC-Whurr; 1992. p. 2-17.

6. Gérard CL. L'enfant dysphasique, 2 ed. Bruxelles: De Boeck Université; 1993.

7. Monfort M, Juárez A. Los niños disfásicos. Madrid: CEPE; 1993.

8. Belinchón M. Las disfasias como dificultades específicas de la adquisición del lenguaje: supuestos, usos y dificultades de un supuesto equívoco. En García JN, ed. Instrucción, aprendizaje y dificultades. Barcelona: Librería Universidad de Barcelona; 1997. p. 231-91.

9. Chevrie-Muller C. Trastornos específicos del desarrollo del lenguaje. En Narbona J, Chevrie-Muller C, eds. El lenguaje del niño. Barcelona: Masson; 1997. p. 249-75.

10. Chevrie-Muller C, Narbona J. Clasificación de los trastornos del lenguaje en el niño. En Narbona J, Chevrie-Muller C, eds. El lenguaje del niño. Barcelona: Masson; 1997. p. 183-7.

11. Landau WM, Goldstein R, Kleffner FR. Congenital aphasia. A clinicopathological study. Neurology 1960; 20: 915-21.

12. Cohen M, Campbell R, Yaghmai F. Neuropathological abnormalities in developmental dysphasia. Ann Neurol 1989; 25: 567-70.

13. Galaburda AM, Rosen GD, Shennan G, Humphreys P. Anatomy of dyslexia: argument against phrenology. In Duane DD, Gray DB, eds. The reading brain. The biological basis of dyslexia. Parkton: York Press; 1991. 
14. Jernigan TL, Hesselink JR, Sowell E, Tallal P. Cerebral structure on magnetic resonance imaging in language and learning impaired children. Arch Neurol 1991; 48: 539-45.

15. Geschwind N, Levitsky W. Human brain: left-right asymmetries in temporal speech region. Science 1968; 161: 186-8.

16. Wada JA, Clarke R, Hamm A. Cerebral hemispheric asymmetry in humans. Arch Neurol 1975; 32: 239-45.

17. Chi JG, Dooling EC, Gilles F. Left-right asymmetries of the temporal speech areas of the human fetus. Arch Neurol 1977; 34: 346-8.

18. Geschwind N, Behan PO. Laterality, hormones and immunity. In Geschwind N, Galaburda AM, eds. Cerebral dominance: the biological foundations. Cambridge: Harvard University Press; 1983. p 211-24.

19. Plante E, Boliek C, Winkievicz A, Erly WK. Elevated androgen, brain development and language/learning disabilities in children with congenital adrenal hyperplasia. Dev Med Child Neurol 1996; 38: 423-37.

20. Lou HC, Henriksen L, Brunh P. Focal cerebral dysfunction in developmental learning disabilities. Lancet 1990; 335: 8-11.

21. Raynaud C, Billard C, Tzoring N, Chiron C, Zilbovizius M, Dulac O, et al. Study of CBF in developmental dysphasia children at rest and during verbal stimulation. J Cereb Blood Flow Metab 1989; 9 (Suppl 1): 323.

22. Tzourio N, Heim A, Zilbovizius M, Gérard C, Mazoyer BM. Abnormal regional CBF response in left-hemisphere of dysphasic children during a language task. Pediatr Neurol 1994; 10: 20-6.

23. Maccario M, Hefferen SJ, Keblused S, Lipinski KA. Developmental dysphasia and electroencephalographic abnormalities. Dev Med Child Neurol 1982; 24: 141-55.

24. Tuchman RF, Rapin I, Shinnar S. Autistic and dysphasic children. II. Epilepsy. Pediatrics 1991; 88, 1219-25.

25. Échenne B, Cheminal R, Rivier F, Negre C, Touchon J, Billiard M. Epileptic electroencephalographic abnormalities and developmental dysphasias: a study of 32 patients. Brain Dev 1992; 14: 216-25.

26. Cheliout-Héraut F, Picard A, Turlan D, Lacert P, de Lattre J. Dysphasies et anomalies paroxystiques. ANAE 1992; 4: 177-84.

27. Duvelleroy-Hommet C, Billard C, Lucas B, Gillet P, Barthez MA, Santini JJ, et al. Sleep EEG and developmental dysphasia. Lack of consistent relationship with paroxysmal EEG activity, during sleep. Neuropediatrics 1995; 26: 14-8.

28. Rapin I. Autistic regression and disintegrative disorder: how important is the role of epilepsy? Semin Pediatr Neurol 1995; 2: 278-85.

29. Deonna T. Trastornos del lenguaje y epilepsia. En Narbona J, Chevrie-Muller C, eds. El lenguaje del niño. Barcelona: Masson; 1997. p. 387-400.

30. Billard C. Neurofisiología y neuroimagen. Sus aplicaciones en la patología del lenguaje del niño. En Narbona J, Chevrie-Muller C, eds. El lenguaje del niño. Barcelona: Masson; 1997. p. 169-80.

31. Picard A, Cheliout-Héraut F, Bouskraoui M, Lemoine M, Lacen P, Delattre J. Sleep EEG and developmental dysphasia. Dev Med Child Neurol 1998; 40: 5959.

32. Tallal P, Piercy M. Developmental aphasia: rate of auditory processing and selective impairment of consonant perception. Neuropsychologia 1974; 12: 8393. 
33. Tallal P, Stark RE, Mellits D. The relationship between auditory temporal analysis and receptive language development: evidence from studies of developmental language disorder. Neuropsychologia 1985; 23: 527-34.

34. Tallal P, Miller SL, Fitch RH. Neurobiological basis of speech: a case for the preeminence of temporal processing. Ann N Y Acad Sci 1993; 682: 27-47.

35. Tallal P, Miller S, Bedi G, Byma G, Wang X, Nagarajan SS, et al. Language comprehension in language-learning impaired children improved with acoustically modified speech. Science 1996; 271: 81-4.

36. Merzenich MM, Jenkins WM, Johnston P, Schereiner C, Miller SL, Tallal P. Temporal processing deficits of language-learning impaired children ameliorated by training. Science 1996; $271: 77-81$.

37. Cowan WM, Fawcett JW, O'Leary DM, Stanfield BB. Regressive events in neurogenesis. Science 1984; 225: 1258-65.

38. Artieda J, Pastor MA. Neurophysiological mechanisms of temporal perception. In Pastor MA, Artieda J, eds. Time, internal clocks and movement. Amsterdam: Elsevier; 1996. p. 1-25.

39. Nichelli P. Time perception measurements in neuropsychology. In Pastor MA, Artieda J, eds. Time, infernal clocks and movement. Amsterdam: Elsevier; 1996. p. 187-204.

40. López-Barneo J. La electricidad cerebral y el lenguaje de las neuronas. En Mora F, ed. El cerebro íntimo. Barcelona: Ariel; 1996. p 28-39.

41. Gathercole SE, Baddeley AD. Phonological memory deficits in language disordered children: is there a causal connection? J Memory Lang 1990; 29: 33660.

42. Crosson B. Subcortical functions in memory and learning. New York: Guildford Press; 1992. p. 111-46.

43. Dayley AT, McKahn GM, Berger MS. The pathophysiology of oral pharyngeal apraxia and mutism following posterior fossa tumor resection in children. $\mathrm{J}$ Neurosurg 1995; 11: 306-10.

44. Koh S, Turkel SB, Baram TZ. Cerebellar mutism in children: report of six cases and potential mechanisms. Pediatr Neurol 1997; 6: 218-9.

45. Jan JE, Kearney S, Groenveld M, Sargent MA, Poskitt KJ. Speech, cognition and imaging studies in congenital ocular motor apraxia (OMA). Dev Med Child Neurol 1998; 40: 95-9.

46. Bishop DVM, North T, Donlan C. Genetic basis of specific language impairment: evidence from a twin study. Dev Med Child Neurol 1995; 37: 5671.

47. Tallal P, Ross R, Curtiss D. Unexpected sex-ratio in families of languagelearning impaired children. Neuropsychologia 1989; 27: 987-98.

48. Tallal P, Ross R, Curtiss S. Familial aggregation in specific language impairment. J Speech Hear Disord 1989; 54: 167-73.

49. Billard C, Toutain A, Loisel ML, Gillet P, Duvelleroy-Hommet C, BarthezCarpentier MA, et al. Dysphasies de développement familiales. Onze cas rapportés dans six families. ANAE 1994; 6: 155-62.

50. Gopnik M, Crago MB. Familial aggregation of a developmental language disorder. Cognition 1991; 39: 1-50.

51. Hurst JA, Baraltser M, Auger E, Graham F, Norell S. An extended family with an inherited speech disorder. Dev Med Child Neurol 1990; 32: 347-55.

52. Van der Lely HK, Stollwerk L. A gramm<atical specific language impairment in children: an autosomal dominant inheritance? Brain Lang 1996; 52: 484-504. 
53. Sudhalter V, Scarborough HS, Cohen IL. Syntactic delay and pragmatic deviance in language of fragile-X males. Am J Med Genet 1993; 38: 493-7.

54. Pembrey M. Genetics and language disorders. In Fletcher P, Hall D, eds. Specific speech and language disorders in children: correlates, characteristics and outcomes. London: AFASIC-Whurr; 1992. p. 51-62.

55. Leonard CM, Williams CH, Nicholls RD, Agee OF, Voeller KK, Honeyman JC, et al. Angelman and Prader-Willi syndromes: a magnetic resonance imaging study of differences in brain structure. Am J Med Genet 1993; 46: 26-33.

56. Bellugi U, Bihrle A, Jernigan T, Trauner D, Doherty S. Neuropsychological and neuroanatomical profile of Williams syndrome. Am J Med Genet 1990; (Suppl 6): $115-25$.

57. Kanniloff-Smith A, Grant J, Bertoud I, Davies M, Howlin P, Udwin O. Language and Williams syndrome: how intact is 'intact'? Child Dev 1997; 68: 246-62.

58. Schiff NB. The influence of deviant maternal input on the development of language during the preschool years. J Speech Hear Res 1979; 22: 581-93.

59. Cross TG, Nienhuys TG, Kirkman M. Parent-child interaction with receptively disabled children: some determinants of maternal speech style. In Nelson K, ed. Children's language. Vol. 5. New York: Gardner Press; 1985. p. 247-89.

60. Hadders-Algra M, Touwen BCL, Hisjes HC. Neurologically deviant newborns: neurological and behavioral development at the age of six years. Dev Med Child Neurol 1986; 28: 569-78.

61. Lindahl E, Michelsson K, Helenius M, Parre M. Neonatal risk factors and later neuro-developmental disturbances. Dev Med Child Neurol 1988; 30: 571-89.

62. Haynes C, Naidoo S. Children with specific speech and language impairment. London: Mac Keith Press; 1991. p. 62-4.

63. Hutcheson GH, Conti-Ramsden G. Qualitative differences in the conversational interactions of SLI children and their younger siblings. In Fletcher P, Hall D, eds. Specific speech and language disorders in children: correlates, characteristics and outcomes. London: AFASIC-Whurr; 1992. p. 18-28.

64. Luoma L, Herrgárd E, Martikainen A, Ahonen T. Speech and language development of children born at 32 weeks' gestation: a 5-year prospective follow-up study. Dev Med Child Neurol 1998; 40: 380-7.

65. Kiessling L, Denckla M, Carlton M. Evidence for differential hernispheric function in children with hemiplegic cerebral palsy. Dev Med Child Neurol 1983; 25: 727-34.

66. Gabari I, Narbona J. Lesión unihemisférica temprana y funciones neuropsicológicas: estudio en 24 hemipléjicos congénitos. En Narbona J, PochOlivé ML, eds. Neuropsychologie infantile: données récentes. Pamplona: Universidad de Navarra; 1987. p. 259-60.

67. Schrager OL. El sistema postural y sus relaciones con las llamadas 'afasias infantiles'. En Bernaldo de Quirós J, ed. Las llamadas afasias infantiles, 2 ed. Buenos Aires: Panamericana; 1975. p. 51-64.

68. Aram DA, Eisele JA. Limits to a hemisphere explanation for specific language impairment. J Speech Hear Res 1994; 37: 824-30. 


\section{TRANSTORNOS ESPECÍFICOS DO DESENVOLVIMENTO DA LINGUAGEM; BASES NEUROBIOLÓGICAS}

\section{RESUMO}

$\mathrm{Na}$ origem das disfasias ou transtornos específicos do desenvolvimento da linguagem (TEDL) se postula actualmente urna predisposção genética, evidenciada por estudos de gémeos, de árvores familiares e de fenotipos peculiares; tal predisposição segue a maior parte das vezes o modelo multifactorial mas também nalgumas familias demonstra-se una transmissão autosómica dominante de incompetência para distintos componentes modulares da linguagem. Os estudos de neuroimnagem podem mostrar alterações da assimetria volumétrica entre os hemisférios cerebrais. Algumos TEDL têm relação fisiopatológica com actividade epiléptica durante o acordado e o sonho não-REM, e o tratamento antiepiléptico, sobretudo com esteroides, se mostrou útil nestos casos. Os sujeitos com TEDL têm uma dificuldade especial para a discriminação subtil de índices temporais, o que convida a establecer uma hipótese que tenga em conta uma possível disfunção de estruturas subcorticais (tálamo-estriadas e cerebelosas) que provavelmente modulam o trabalho do córtex na integração fonológica.

\section{PALAVRAS CLAVE}

Discriminação temporal. Disfasia. Epilepsia. Genética. Transtorno do desenvolvimento da linguagem.

\section{THE NEUROBIOLOGICAL BACKGROUND OF SPECIFIC DEVELOPMENTAL LANGUAGE DISORDERS}

\section{SUMMARY}

Studies of twins, familial aggregates and particular phenolypic conditions have shown an inherited basis for some dysphasias or specific developmental language impairments (SLI). This predisposition is usually multifactorial but the analysis of some families allows to postulate an autosomal dominant transmission of deficits in specific modular aspects of linguistic competentes. Moreover, neuroimaging studies have shown modifications of normal volumetric interhemispheric asymmetries, and in group of SLI with receptive prominent disorder coexist epileptiform activity in wakefulness and nonREM sleep EEG; in some of these cases, antiepileptic drugs, specially steroids, can significantly ameliorate the language processing. As many patients with SLI have a difficulty for discrimination of subtle temporal indices, a hypothesis can also be made of a dysfunction in various subcortical structures (thalamus, basal ganglia, cerebellum) modulating the cerebral cortex in phonological processing.

\section{KEY WORDS}

Dysphasia. Epilepsy. Genetics. Specific language disorders. Temporal discrimination. 
Tabla I. Hallazgos neurobiológicos en TEDL.

Trastornos estructurales y funcionales encefálicos

Alteración de las asimetrías planimétricas interhemisféricas

Displasias corticales perisilvianas

Hipoplasia de núcleo caudado y tálamo

Displasia cerebelosa

Estudios de función cerebral

Hipoperfusión bitemporal (SPECT)

Descargas paroxísticas persistentes en EEG de sueño

Bases genéticas

Estudios de concordancia en gemelos

Incidencia familiar (modelos: multifactorial y autosómico dominante)

Fenotipos con peculiar trastorno de lenguaje y deficiencia mental

Síndrome frágil X

Síndrome de Williams

Síndrome de Angelman 